\title{
Standardized Karyotype and Idiogram of Bengal Monitor Lizard, Varanus bengalensis (Squamata, Varanidae)
}

\author{
Isara Patawang ${ }^{1}$, Alongklod Tanomtong ${ }^{2,5 *}$, Nuntaporn Getlekha ${ }^{2,5}$, \\ Sumalee Phimphan ${ }^{2,5}$, Krit Pinthong ${ }^{3}$ and Lamyai Neeratanaphan ${ }^{4,5}$
}

\footnotetext{
${ }^{1}$ Department of Biology, Faculty of Science, Chiang Mai University, Chiang Mai, Muang 50200, Thailand

${ }^{2}$ Department of Biology, Faculty of Science, Khon Kaen University, Khon Kaen, Muang 40002, Thailand

${ }^{3}$ Department of Fundamental Science, Faculty of Science and Technology, Surindra Rajabhat University, Surin, Muang 32000, Thailand

${ }^{4}$ Department of Environmental Science, Faculty of Science, Khon Kaen University, Khon Kaen, Muang 40002, Thailand

${ }^{5}$ Research Group on Toxic Substances in Livestock and Aquatic Animals, Khon Kaen University, Khon Kaen, Muang 40002, Thailand
}

Received July 10, 2015; accepted November 10, 2015

\begin{abstract}
Summary The standardized karyotype and idiogram of the Bengal monitor lizard (Varanus bengalensis) from the Kalasin Province of Thailand were studied. Blood samples were taken from male and female lizards. The standard T-lymphocyte cell was cultured at $26^{\circ} \mathrm{C}$ for $96 \mathrm{~h}$. The mitotic chromosomes were harvested by colchicine-hypotonic-fixation-air drying technique. Conventional staining, Ag-NOR banding and C-banding techniques were applied to stain the chromosome with $20 \%$ Giemsa's solution. The results showed that the number of diploid chromosome was $2 n=40$, while the fundamental number (NF) was 54 in males and 55 in females. The types of macrochromosomes were 4 large metacentric, 4 small metacentric, 6 small submetacentric, 2 small telocentric macrochromosomes and 24 microchromosomes. The $\mathrm{Z}$ chromosome is the small telocentric chromosome, while the $\mathrm{W}$ chromosome is the small metacentric chromosome. In addition, the short arm pericentromeric region of chromosome pair 1 showed clearly observable nucleolar organizer regions (NORs). This data is the first report on polymorphism of NORs in $V$. bengalensis. The result showed that the heteromorphic of one male had a different size of NORs of chromosome pair 1 (1a1b), while one female had an equal size of both chromosome pair 1 with a homomorphic (1a1a). The karyotype formula is as follows:
\end{abstract}

$2 n(40)=\mathrm{L}_{4}^{\mathrm{m}}+\mathrm{S}_{4}^{\mathrm{m}}+\mathrm{S}_{6}^{\mathrm{sm}}+\mathrm{S}_{2}^{\mathrm{t}}+\mathrm{Z}(\mathrm{t}) \mathrm{W}(\mathrm{m})+22$ microchromosomes

Key words Bengal monitor lizard, Varanus bengalensis, Karyotype, Chromosome.

The Bengal monitor lizard, Varanus bengalensis (Gray 1831) (Fig. 1), is a member of class Reptilia, order Squamata, suborder Lacertilia, superfamily Varanoidea and family Varanidae. All living monitor lizards are placed in a single genus Varanus, generally considered to be monophyletic. At this time, there are 46 species that are found in Africa, Asia and Australia. Four species of the genus Varanus have been recorded in Thailand: $V$. bengalensis, $V$. salvator (Laurenti 1768), $V$. rudicollis (Gray 1845) and V. dumerilii (Schlegel 1839) (Bennett 1998, Lauprasert and Thirakhupt 2001, Pianka 1995).

The Bengal monitor lizard is a member of the monitor or Varanid lizards group, native to Afghanistan, Pakistan, India, Sri Lanka, Bangladesh, Nepal, southern China, Myanmar, Laos, Vietnam, Cambodia and Thailand. The visual characteristics of $V$. bengalensis are a slender body, snout somewhat elongated, nostrils nearer the eyes than the snout-tip, tail flattened, unicoloured or with a less distinct pattern, chin and throat with black spots, venter cream or yellow. The lizard's diet consists of insects, snails, frogs, small mammals, birds, lizards and snakes (Das 2010) (Fig. 1).

Lizards from the family Varanidae belong to the group of squamates for which the karyotypes appear highly conserved. Until now, there were only three previous cytogenetic studies of $V$. bengalensis according to the cytogenetic studies by Dutt (1968), Singh et al. (1970) and De Smet (1981). The present study is the first report on the chromosomal characteristics of $V$. bengalensis using Ag-NOR banding and C-banding techniques. The results of this study enhances cytogenetic information and enables future comprehensive studies to be conducted on taxonomy and evolutionary relationships.

\footnotetext{
* Corresponding author, e-mail: tanomtong@hotmail.com

DOI: $10.1508 /$ cytologia. 82.75
} 


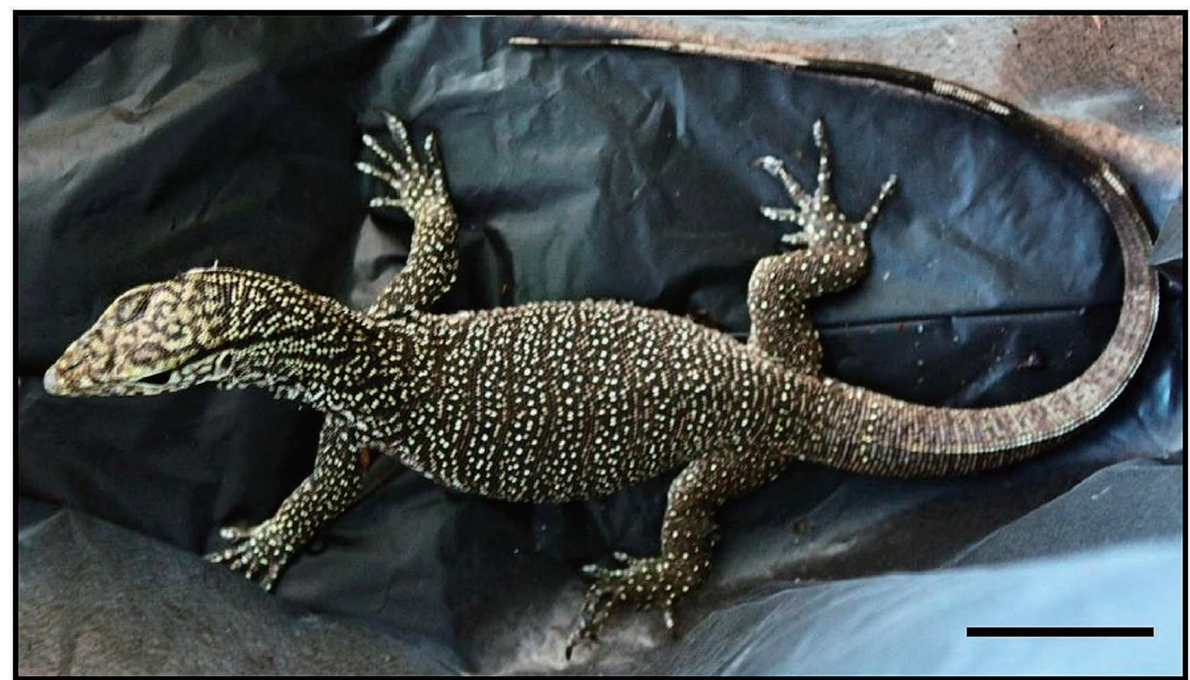

Fig. 1. General characteristics of the Bengal monitor, Varanus bengalensis (Varanidae, Squamata) from Kalasin Province of Northeastern Thailand; scale bar indicates $10 \mathrm{~cm}$.
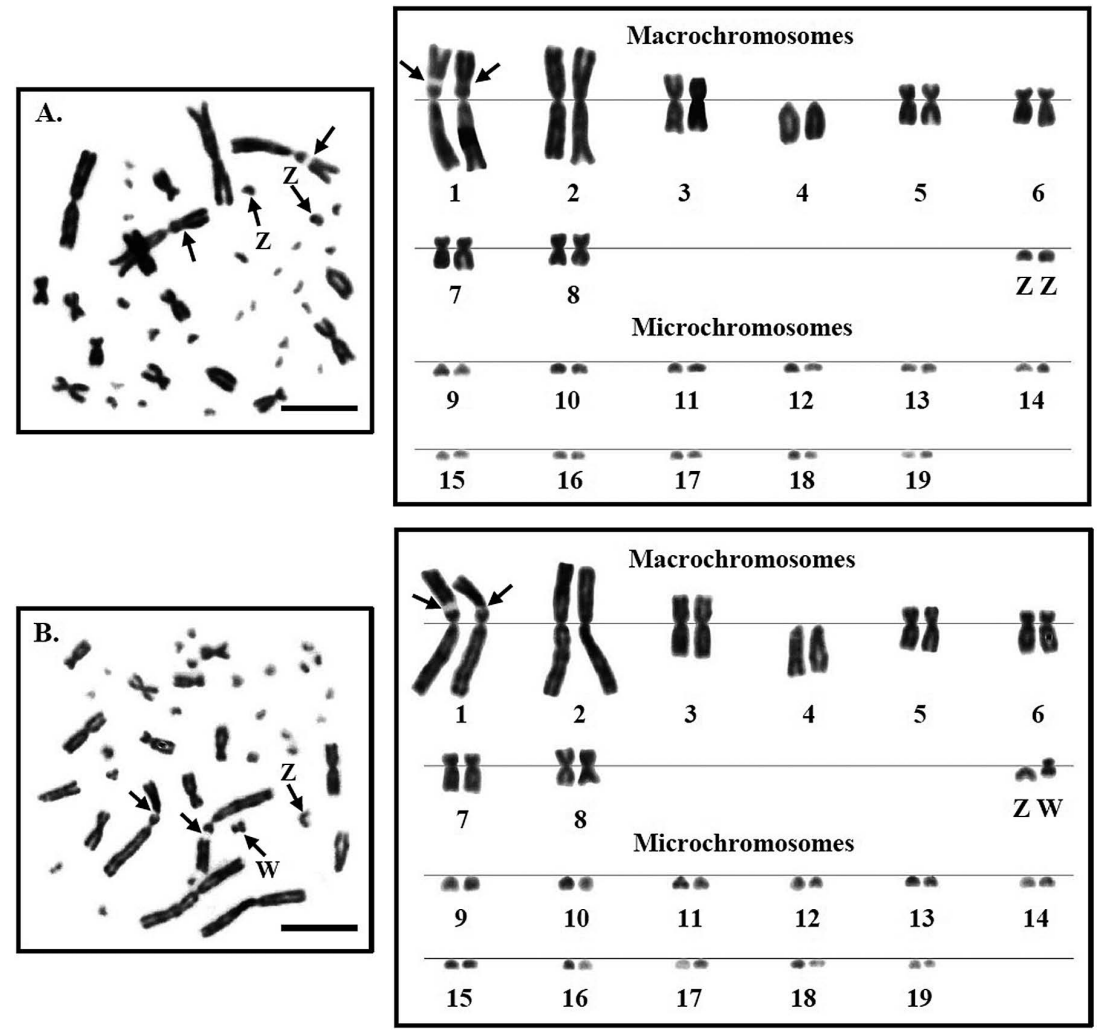

Fig. 2. The metaphase chromosome plates and karyotypes of male (A) and female (B) Bengal monitors (Varanus bengalensis), $2 n=40$, by conventional staining technique. The secondary constrictions appear on the subcentromeric on the short arm of the largest metacentric chromosome pair 1 (arrows). Scale bar indicates $5 \mu \mathrm{m}$.

Materials and methods

\section{Blood samples and cell cultures}

Blood samples from one male and one female $V$. bengalensis were maintained in the Kalasin Province of Thailand. The samples were collected from the blood vein using an aseptic technique, and kept on ice in $5 \mathrm{~mL}$ vacuum tubes coated with heparin to prevent blood clot- ting. After cooling, $0.5 \mathrm{~mL}$ of whole blood were cultured in $5 \mathrm{~mL}$ of RPMI 1640 medium supplemented with $2 \%$ phytohemagglutinin (PHA) as a mitogen at $26^{\circ} \mathrm{C}, 5 \%$ $\mathrm{CO}_{2}$. The cultured bottle was loosely capped and regularly shaken twice a day, in the morning and evening. After a $96 \mathrm{~h}$ incubation, colchicine was introduced and mixed for further incubation for $30 \mathrm{~min}$. 

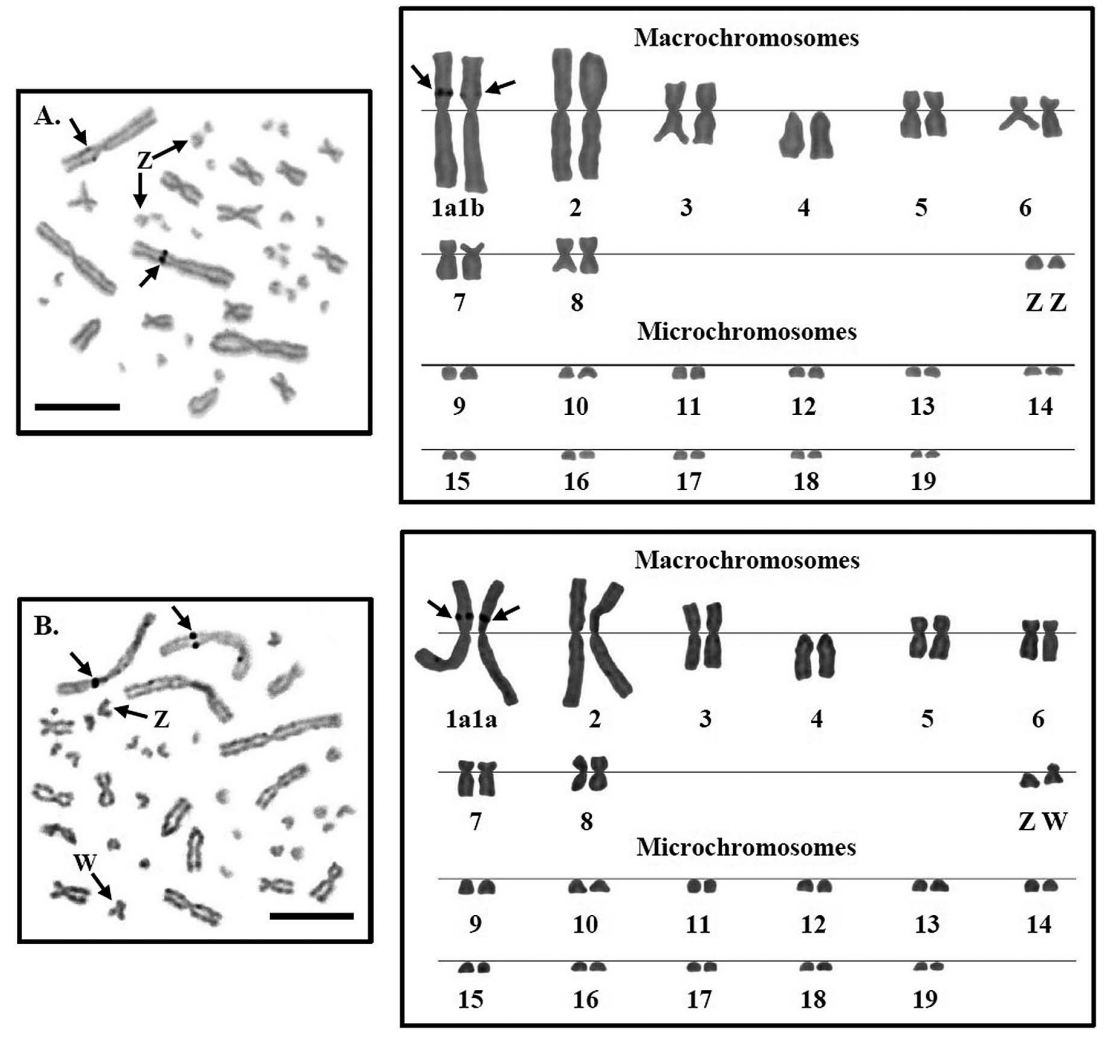

Fig. 3. The metaphase chromosome plates and karyotypes of male (A) and female (B) Bengal monitors (Varanus bengalensis), $2 n=40$, by Ag-NOR banding technique. The result showed that a heteromorphic of one male had a different size of NORs of chromosome pair 1 (1alb), while one male had an equal size of both chromosome pair 1 with a homomorphic (1ala). Scale bar indicates $5 \mu \mathrm{m}$.
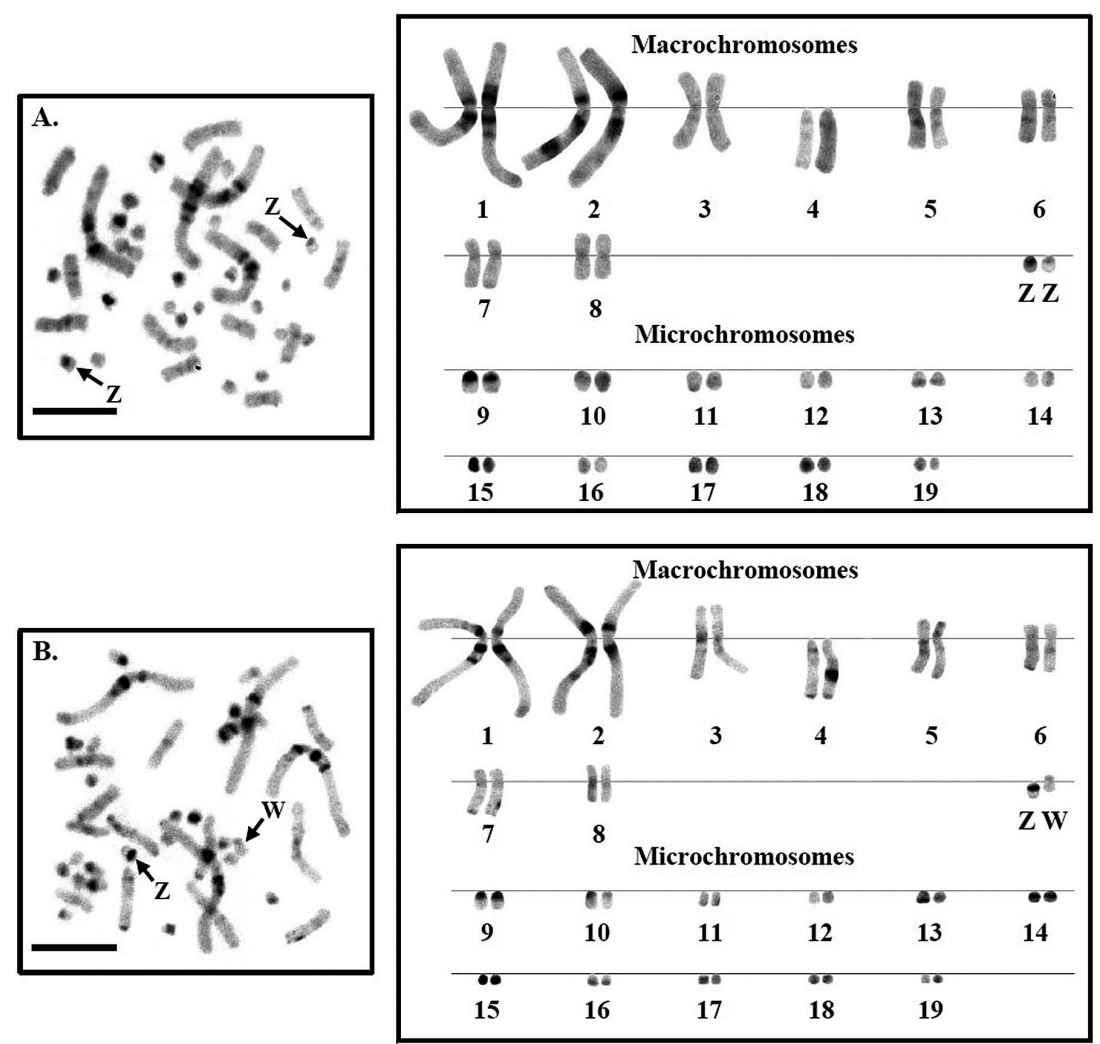

Fig. 4. The metaphase chromosome plates and karyotypes of male (A) and female (B) Bengal monitors (Varanus bengalensis), $2 n=40$, by C-banding technique. Scale bar indicates $5 \mu \mathrm{m}$. 
Table 1. Review of cytogenetic reports of lizards in family Varanidae (Reptilia, Squamata).

\begin{tabular}{|c|c|c|c|c|c|}
\hline Species & $2 n$ & $\operatorname{mac}$ & $\mathrm{mi}$ & NORs & Reference \\
\hline Varanus acanthurus & $40(\mathrm{ZW})$ & $16(14 b i+2 t)$ & 24 & $1^{\text {st }}$ & King et al. (1982) \\
\hline$V$. albigularis & $40(\mathrm{ZW})$ & $16(8 b i+8 t)$ & 24 & $1^{\text {st }}$ & King and King (1975) \\
\hline \multirow[t]{4}{*}{$V$. bengalensis } & 40 & $16(8 b i+8 t)$ & 24 & - & Dutt (1968) \\
\hline & 40 & $16(14 b i+2 t)$ & 24 & - & Singh et al. (1970) \\
\hline & 40 & $16(16 b i)$ & 24 & - & De Smet (1981) \\
\hline & $40(\mathrm{ZW})$ & $16(14 b i+2 t)$ & 24 & $1^{\text {st }}$ & Present study \\
\hline V. exanthematicus & 40 & $16(8 b i+8 t)$ & 24 & - & De Smet (1981) \\
\hline$V$. flavescens & 40 & $16(14 b i+2 t)$ & 24 & - & Singh et al. (1970) \\
\hline$V$. giganteus & 40 & $16(10 b i+6 t)$ & 24 & $1^{\text {st }}$ & King and King (1975) \\
\hline$V$. gilleni & 40 & $16(14 b i+2 t)$ & 24 & $1^{\text {st }}$ & King and King (1975) \\
\hline \multirow[t]{2}{*}{$V$. gouldii } & 40 & $16(8 b i+8 t)$ & 24 & - & Matthey (1931) \\
\hline & 40 & $16(10 b i+6 t)$ & 24 & $1^{\text {st }}$ & King and King (1975) \\
\hline \multirow[t]{2}{*}{ V. griseus } & 40 & $16(16 \mathrm{bi})$ & 24 & $1^{\text {st }}$ & King and King (1975) \\
\hline & 40 & $16(14 b i+2 t)$ & 24 & $1^{\text {st }}$ & De Smet (1981) \\
\hline V. indicus & 40 & $16(14 b i+2 t)$ & 24 & $1^{\text {st }}$ & King and King (1975) \\
\hline$V$. mertensi & 40 & $16(10 b i+6 t)$ & 24 & $1^{\text {st }}$ & King and King (1975) \\
\hline V.nebulosus & 40 & 16 (16bi) & 24 & $1^{\text {st }}$ & King and King (1975) \\
\hline \multirow[t]{2}{*}{ V. niloticus } & $40(\mathrm{ZW})$ & $16(8 b i+8 t)$ & 24 & $1^{\text {st }}$ & King and King (1975) \\
\hline & 40 & $16(8 b i+8 t)$ & 24 & $1^{\text {st }}$ & Porter et al. (1994) \\
\hline$V$. rosenbergi & 40 & $16(10 b i+6 t)$ & 24 & $1^{\text {st }}$ & King and King (1975) \\
\hline V. rudicollis & 40 & $16(14 b i+2 t)$ & 24 & - & Gorman and Gress (1970) \\
\hline \multirow[t]{2}{*}{ V. salvator } & 40 & 16 (16bi) & 24 & $1^{\text {st }}$ & King and King (1975) \\
\hline & 40 & $16(14 b i+2 t)$ & 24 & - & De Smet (1981) \\
\hline V. scalaris & 40 & $16(14 b i+2 t)$ & 24 & $1^{\text {st }}$ & King and King (1975) \\
\hline$V$. semiremex & 40 & $16(14 b i+2 t)$ & 24 & $1^{\text {st }}$ & King and King (1975) \\
\hline V. spenceri & 40 & $16(10 b i+6 t)$ & 24 & $1^{\text {st }}$ & King and King (1975) \\
\hline V. storri & 40 & $16(14 b i+2 t)$ & 24 & $1^{\text {st }}$ & King and King (1975) \\
\hline V. tristis & 40 & $16(14 b i+2 t)$ & 24 & $1^{\mathrm{st}}, 7^{\mathrm{th}}$ & King and King (1975) \\
\hline$V$. varius & $40(\mathrm{ZW})$ & $16(14 b i+2 t)$ & 24 & $1^{\text {st }}$ & King and King (1975) \\
\hline
\end{tabular}

Remarks: $2 n=$ diploid chromosome, $\mathrm{mac}=$ macrochromosome, $\mathrm{mi}=$ microchromosome, bi=bi-arms chromosome, $\mathrm{t}=$ telocentric chromosome and $-=$ not available.

Table 2. Mean length (L) of the short arm chromosome (Ls), long arm chromosome (L1), and total arm chromosome (LT), relative length (RL), centromeric index (CI) and standard deviation (SD) of RL, CI from metaphase chromosomes in 10 cells of male Bengal monitor (Varanus bengalensis), $2 n=40$.

\begin{tabular}{|c|c|c|c|c|c|c|c|}
\hline Chro. pair & Ls & $\mathrm{Ll}$ & LT & $\mathrm{CI} \pm \mathrm{SD}$ & $\mathrm{RL} \pm \mathrm{SD}$ & Chro. type & Chro. size \\
\hline $1 *$ & 7.331 & 9.522 & 16.853 & $0.565 \pm 0.005$ & $0.195 \pm 0.003$ & Metacentric & Large \\
\hline 2 & 7.022 & 8.431 & 15.453 & $0.546 \pm 0.004$ & $0.179 \pm 0.003$ & Metacentric & Large \\
\hline 3 & 3.612 & 4.064 & 7.676 & $0.529 \pm 0.002$ & $0.089 \pm 0.002$ & Metacentric & Small \\
\hline 4 & 0.000 & 5.752 & 5.752 & $1.000 \pm 0.000$ & $0.067 \pm 0.005$ & Telocentric & Small \\
\hline 5 & 2.271 & 3.461 & 5.732 & $0.604 \pm 0.006$ & $0.066 \pm 0.003$ & Submetacentric & Small \\
\hline 6 & 1.885 & 3.622 & 5.507 & $0.658 \pm 0.003$ & $0.064 \pm 0.004$ & Submetacentric & Small \\
\hline 7 & 1.833 & 2.762 & 4.595 & $0.601 \pm 0.002$ & $0.053 \pm 0.003$ & Submetacentric & Small \\
\hline 8 & 2.111 & 2.462 & 4.573 & $0.538 \pm 0.005$ & $0.053 \pm 0.004$ & Metacentric & Small \\
\hline 9 & - & - & 2.201 & - & $0.025 \pm 0.002$ & \multicolumn{2}{|c|}{ Microchromosome } \\
\hline 10 & - & - & 2.002 & - & $0.023 \pm 0.002$ & \multicolumn{2}{|c|}{ Microchromosome } \\
\hline 11 & - & - & 1.701 & - & $0.020 \pm 0.001$ & \multicolumn{2}{|c|}{ Microchromosome } \\
\hline 12 & - & - & 1.653 & - & $0.019 \pm 0.003$ & \multicolumn{2}{|c|}{ Microchromosome } \\
\hline 13 & - & - & 1.634 & - & $0.019 \pm 0.003$ & \multicolumn{2}{|c|}{ Microchromosome } \\
\hline 14 & - & - & 1.603 & - & $0.019 \pm 0.002$ & \multicolumn{2}{|c|}{ Microchromosome } \\
\hline 15 & - & - & 1.556 & - & $0.018 \pm 0.002$ & \multicolumn{2}{|c|}{ Microchromosome } \\
\hline 16 & - & - & 1.537 & - & $0.018 \pm 0.002$ & \multicolumn{2}{|c|}{ Microchromosome } \\
\hline 17 & - & - & 1.482 & - & $0.017 \pm 0.003$ & \multicolumn{2}{|c|}{ Microchromosome } \\
\hline 18 & - & - & 1.431 & - & $0.017 \pm 0.003$ & \multicolumn{2}{|c|}{ Microchromosome } \\
\hline 19 & - & - & 1.300 & - & $0.015 \pm 0.001$ & \multicolumn{2}{|c|}{ Microchromosome } \\
\hline $\mathrm{Z}$ & 0.000 & 2.17 & 2.170 & $1.000 \pm 0.000$ & $0.025 \pm 0.003$ & Telocentric & Small \\
\hline
\end{tabular}

Remarks: *=Nucleolar organizer region, (NOR) and chro. $=$ chromosome

\section{Cell harvest}

After colchicine incubation, the blood mixture was centrifuged at $1200 \mathrm{rpm}$ for $10 \mathrm{~min}$. After discarding the supernatant, the cells were treated with $10 \mathrm{~mL}$ of hypo- tonic solution $(0.075 \mathrm{M} \mathrm{KCl})$ and the cells were incubated at $26^{\circ} \mathrm{C}$ for $30 \mathrm{~min}$. The cells then were centrifuged and the supernatant was discarded. Fresh cool fixative (3 methanol:1 acetic acid) was used to fix the cells by 
Table 3. Mean length (L) of the short arm chromosome (Ls), long arm chromosome (L1), and total arm chromosome (LT), relative length (RL), centromeric index (CI) and standard deviation (SD) of RL, CI from metaphase chromosomes in 10 cells of female Bengal monitor (Varanus bengalensis), $2 n=40$.

\begin{tabular}{|c|c|c|c|c|c|c|c|}
\hline Chro. pair & Ls & $\mathrm{Ll}$ & LT & $\mathrm{CI} \pm \mathrm{SD}$ & $\mathrm{RL} \pm \mathrm{SD}$ & Chro. type & Chro. size \\
\hline $1 *$ & 8.302 & 10.761 & 19.063 & $0.564 \pm 0.005$ & $0.185 \pm 0.003$ & Metacentric & Large \\
\hline 2 & 8.212 & 9.963 & 18.175 & $0.548 \pm 0.003$ & $0.176 \pm 0.003$ & Metacentric & Large \\
\hline 3 & 4.031 & 4.864 & 8.895 & $0.547 \pm 0.004$ & $0.086 \pm 0.004$ & Metacentric & Small \\
\hline 4 & 0.000 & 7.270 & 7.270 & $1.000 \pm 0.000$ & $0.070 \pm 0.001$ & Telocentric & Small \\
\hline 5 & 2.385 & 3.911 & 6.296 & $0.621 \pm 0.005$ & $0.061 \pm 0.002$ & Submetacentric & Small \\
\hline 6 & 2.077 & 4.205 & 6.282 & $0.669 \pm 0.004$ & $0.061 \pm 0.001$ & Submetacentric & Small \\
\hline 7 & 1.891 & 3.683 & 5.574 & $0.661 \pm 0.004$ & $0.054 \pm 0.003$ & Submetacentric & Small \\
\hline 8 & 2.593 & 2.792 & 5.385 & $0.518 \pm 0.006$ & $0.052 \pm 0.003$ & Metacentric & Small \\
\hline 9 & - & - & 2.370 & - & $0.023 \pm 0.003$ & \multicolumn{2}{|c|}{ Microchromosome } \\
\hline 10 & - & - & 2.301 & - & $0.022 \pm 0.002$ & \multicolumn{2}{|c|}{ Microchromosome } \\
\hline 11 & - & - & 2.203 & - & $0.021 \pm 0.002$ & \multicolumn{2}{|c|}{ Microchromosome } \\
\hline 12 & - & - & 2.054 & - & $0.020 \pm 0.004$ & \multicolumn{2}{|c|}{ Microchromosome } \\
\hline 13 & - & - & 1.931 & - & $0.019 \pm 0.002$ & \multicolumn{2}{|c|}{ Microchromosome } \\
\hline 14 & - & - & 1.866 & - & $0.018 \pm 0.002$ & \multicolumn{2}{|c|}{ Microchromosome } \\
\hline 15 & - & - & 1.780 & - & $0.017 \pm 0.002$ & \multicolumn{2}{|c|}{ Microchromosome } \\
\hline 16 & - & - & 1.707 & - & $0.017 \pm 0.004$ & \multicolumn{2}{|c|}{ Microchromosome } \\
\hline 17 & - & - & 1.621 & - & $0.016 \pm 0.003$ & \multicolumn{2}{|c|}{ Microchromosome } \\
\hline 18 & - & - & 1.563 & - & $0.015 \pm 0.002$ & \multicolumn{2}{|c|}{ Microchromosome } \\
\hline 19 & - & - & 1.453 & - & $0.014 \pm 0.001$ & \multicolumn{2}{|c|}{ Microchromosome } \\
\hline $\mathrm{Z}$ & 0.000 & 2.620 & 2.620 & $1.000 \pm 0.000$ & $0.025 \pm 0.002$ & Telocentric & Small \\
\hline W & 1.161 & 1.720 & 2.881 & $0.597 \pm 0.005$ & $0.028 \pm 0.004$ & Metacentric & Small \\
\hline
\end{tabular}

Remarks: *=Nucleolar organizer region, $(\mathrm{NOR})$ and chro. $=$ chromosome.

gradually adding it up to $8 \mathrm{~mL}$. After centrifugation, the fixation was repeatedly done until the supernatant was clear. The cells were added to $1 \mathrm{~mL}$ fixative by dropping onto a clean cold slide and then dried by air-dry technique (Kasiroek et al. 2017). The slide was conventionally stained with $20 \%$ Giemsa's solution for $30 \mathrm{~min}$ (Chooseangjaew et al. 2017).

\section{C-banding method}

The slides were heated at $60^{\circ} \mathrm{C}$ for two to three days, soaked in $0.2 \mathrm{~N} \mathrm{HCl}$ for $10-15 \mathrm{~min}$, rinsed with distilled water, then soaked in $0.05 \mathrm{~N} \mathrm{Ba}(\mathrm{OH})_{2}$ for $15 \mathrm{~min}$ at $37^{\circ} \mathrm{C}$ and rinsed with distilled water at $60^{\circ} \mathrm{C}$. After that, the slide was soaked in $2 \mathrm{X} \mathrm{SSC}$ at $60^{\circ} \mathrm{C}$ for one to two hours. The slide was then stained with $20 \%$ Giemsa's solution for $30 \mathrm{~min}$ (Rooney 2001, Patawang and Tanomtong 2017).

\section{Ag-NOR banding method}

Two drops of $50 \%$ silver nitrate and $50 \%$ gelatin were added on to the slides, respectively. They were then sealed with cover glasses and incubated at $60^{\circ} \mathrm{C}$ for three hours. After that, the slides were soaked in distilled water until the cover glasses were separated. The slide was stained with $20 \%$ Giemsa's solution for one minute (Howell and Black 1980, Sangpakdee et al. 2017).

\section{Results and discussion}

Conventional staining of the karyotypes identified the diploid number of chromosomes for $V$. bengalensis to be $2 n=40$ (Fig. 2). These karyotypes all consist of
16 macrochromosomes (14 bi-arms chromosomes and 2 mono-arm chromosomes) and 24 microchromosomes. We found the total chromosome number of macrochromosomes and microchromosomes and the morphologies of macrochromosomes of $V$. bengalensis in this study to be identical to those reported previously, confirming that at a gross morphological level, the karyotypes of Varanus species are conservative. Karyotypes have been reported for 22 Varanus species. All species examined so far have an identical chromosome number, $2 n=40$, consisting of 16 macrochromosomes and 24 microchromosomes (Table 1), indicating that the karyotypes at this level are highly conserved in this family. This high conservation of karyotypes makes varanid lizards an excellent model to examine the fine scale molecular evolution of DNA sequences on some local chromosomal regions (Matsubara et al. 2014).

$V$. bengalensis has three types of macrochromosomes, which are 8 metacentric (four large and four small chromosomes), 6 submetacentric and 2 telocentric chromosomes (Table 2, 3). Different chromosomal features were reported by De Smet (1981), which consisted of 16 biarms and 24 uni-arms macrochromosomes. The microchromosomes in the $V$. bengalensis are 24 in number and all of them are nearly the same size. They behave like macrochromosomes but their small size inhibits critical analysis.

Current knowledge shows that the sex-chromosome system of the $V$. bengalensis is $\mathrm{ZW}$, where the $\mathrm{Z}$ and $\mathrm{W}$ are classified as small telocentric and small metacentric chromosomes, respectively. The sex chromosomes are identified in four species ( $V$. acanthurus, $V$. albigularis, 

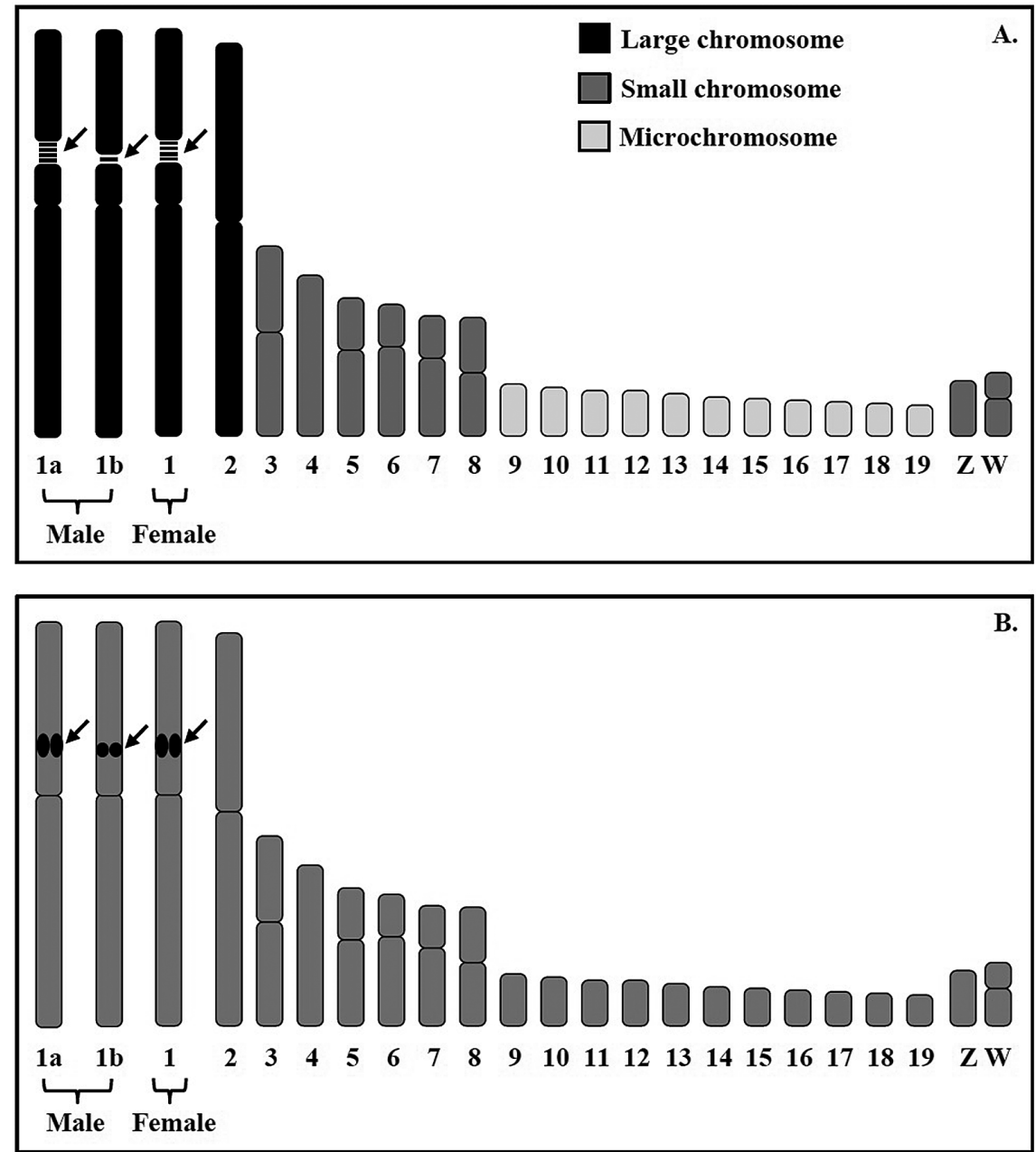

Fig. 5. The standardized idiogram of Bengal monitor (Varanus bengalensis), $2 n=40$, by conventional staining (A) and Ag-NOR banding techniques (B); arrows indicate nucleolar organizer regions (NORs).

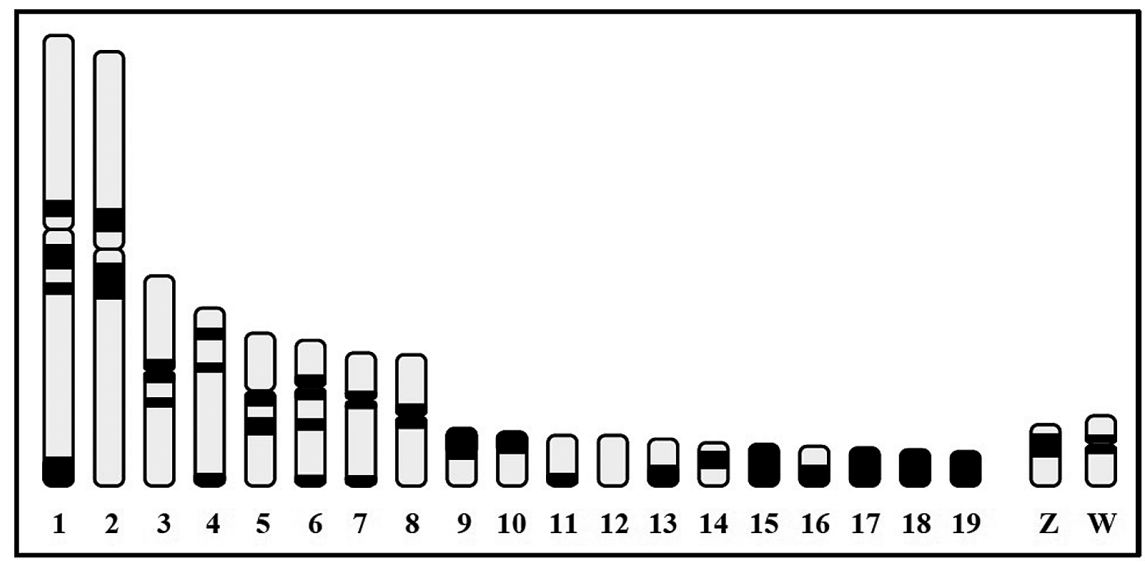

Fig. 6. The standardized idiogram of Bengal monitor (Varanus bengalensis), $2 n=40$, by C-banding technique.

$V$. niloticus and $V$. varius). All four species have ZZ/ZW micro sex chromosomes in which the $\mathrm{W}$ is distinctively larger than other microchromosomes. The sex chromosomes of the many remaining species are unknown, and the molecular composition and constitution of the W chromosomes are yet to be investigated in any species (Matsubara et al. 2014).
The short arm pericentromeric region of chromosome pair 1 showed clearly observable nucleolar organizer regions (NORs) (Fig. 3). This is the first report on polymorphism of NORs in the $V$. bengalensis. The result showed that the heteromorphic of one male had a different size of NORs of chromosome pair 1 (1a1b), while one female had an equal size of both chromosome 

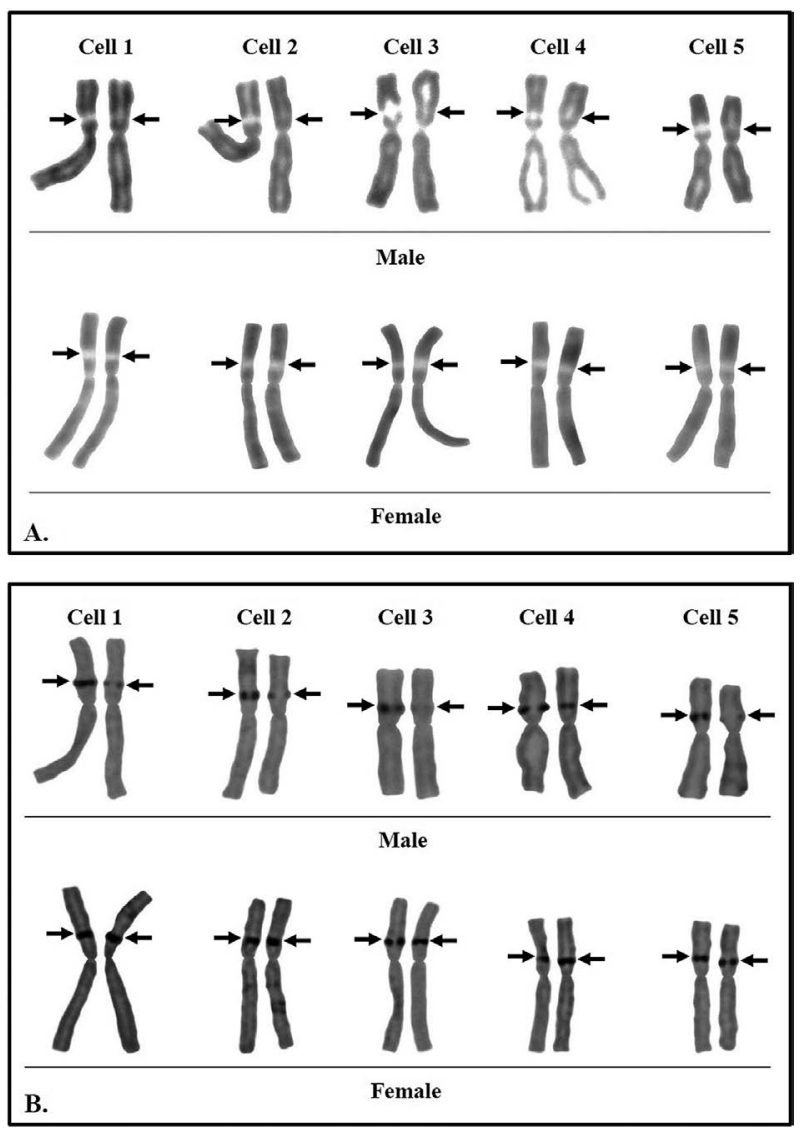

Fig. 7. The comparison of secondary constriction/nucleolar organizer region on largest metacentric chromosome pair 1 between male (heteromorphic, 1a1b) and female (homomorphic, 1a1a) Bengal monitors (Varanus bengalensis) by conventional staining (A) and NOR-banding (B) techniques.

pair 1 with the homomorphic (1ala) (Figs. 5, 7). Porter et al. (1994) found that the ribosomal genes (NORs) of the $V$. niloticus are found near the centromere of pair 1, which is a large metacentric chromosome. The region of hybridization corresponds with a distinct secondary constriction, which King and King (1975) correctly interpreted as the nucleolus organizer. The secondary constriction is found near the centromere of chromosome pair 1 in all karyotyped species of the genus (King and King 1975). Assuming that rDNA can be accurately located by the secondary constriction, it would appear that the ribosomal genes have remained in the same location throughout the evolution of genus Varanus. The proximal location of NORs in varanid chromosomes is unusual. Other squamate reptiles typically have the sites of NORs located near the chromosome ends.

The C-banding can provide a dark region on the chromosome, which represents the constitutive heterochromatin of the chromosome that can be found at all centromeres and some telomere of normal chromosomes. C-bands were detected at the centromeric regions of chromosome pairs 3, 5-8 (macrochromosomes), 9-10, $15,17-19$, and $\mathrm{W}$ (microchromosomes); on interstitial regions of chromosome pairs 1-6 (macrochromosomes),
14, and $\mathrm{Z}$ (microchromosomes); and on the telomeric regions of chromosome pairs 1, 4, 6-7 (macrochromosomes), 11, 13, and 16 (microchromosomes) (Fig. 4).

$V$. bengalensis demonstrated that the chromosome marker is the chromosome pair 1, which is the largest metacentric chromosome. The important karyotype feature of the $V$. bengalensis is the asymmetrical karyotype, which was found in three types of chromosomes (metacentric, submetacentric and telocentric chromosomes). The largest chromosome is eight times larger than the smallest chromosomes. Figures 5, 6 show the idiograms from conventional staining, Ag-NOR banding and Cbanding techniques. The karyotype formula could be deduced as:

$$
\begin{array}{r}
2 n(40)=\mathrm{L}_{4}^{\mathrm{m}}+\mathrm{S}_{4}^{\mathrm{m}}+\mathrm{S}_{6}^{\mathrm{sm}}+\mathrm{S}_{2}^{\mathrm{t}}+\mathrm{Z}(\mathrm{t}) \mathrm{W}(\mathrm{m}) \\
+22 \text { microchromosomes } \\
\text { Acknowledgements }
\end{array}
$$

This work was financially supported by the Research Group on Toxic Substances in Livestock and Aquatic Animals, Khon Kaen University. We would like to thank the laboratory of animal cytogenetic group in the Department of Biology, Faculty of Science, Khon Kaen University and the laboratory of Department of Biology, Faculty of Science, Chiang Mai University for available help.

\section{References}

Bennett, D. 1998. Monitor Lizards: Natural History, Biology and Husbandry. Warlich Druck Press, Meckenheim.

Chooseangjaew, S., Tanyaros, S., Maneechot, N., Buasriyot, P., Getlekha, N. and Tanomtong, A. 2017. Chromosomal characteristics of the tropical oyster, Crassostrea belcheri Sowerby, 1871 (Ostreoida, Ostreidae) by conventional and Ag-NOR banding techniques. Cytologia 82: 3-8.

Das, I. 2010. A Field Guide to the Reptiles of South-East Asia. New Holland Publishers, Chatswood.

De Smet, W. H. O. 1981. Description of the orsein stained karyotypes of 136 lizard species (Lacertilia, Reptilia) belonging to the families Teiidae, Scincidae, Lacertidae, Cordylidae and Varanidae (Austarchoglossa). Acta Zool. Pathol. Antverp. 76: 407-420.

Dutt, K. 1968. A karyotype study of Varanus monitor Linn. Caryologia 21: 1-10.

Gorman, G. C. and Gress, F. 1970. Chromosome cytology of four boid snakes and a varanid lizard, with comments on the cytosystematics of primitive snakes. Herpetologica 26: 308-317.

Howell, W. M. and Black, D. A. 1980. Controlled silver-staining of nucleolus organizer regions with a protective colloidal developer: A 1-step method. Experientia 36: 1014-1015.

Kasiroek, W., Indananda, C., Luangoon, N., Pinthong, K., Supiwong, W. and Tanomtong, A. 2017. First chromosome analysis of the humpback cardinalfish, Fibramia lateralis (Perciformes, Apogonidae). Cytologia 82: 9-15.

King, M. and King, D. 1975. Chromosomal evolution in the lizard genus Varanus (Reptilia). Aust. J. Biol. Sci. 28: 89-108.

King, M., Mengden, G. A. and King, D. 1982. A pericentric inversion polymorphism and a ZZ/ZW sex-chromosome system in Varanus acanthurus Boulenger analyzed by G- and C-banding and Ag staining. Genetica 58: 39-45. 
Lauprasert, K. and Thirakhupt, K. 2001. Species diversity, distribution and proposed status of Monitor lizards (family Varanidae) in Southern Thailand. The Natural History Journal of Chulalongkorn University 1: 39-46.

Matsubara, K., Sarre, S. D., Georges, A., Matsuda, Y., Graves, J. A. M. and Ezaz, T. 2014. Highly differentiated ZW sex microchromosomes in the Australian Varanus species evolved through rapid amplification of repetitive sequences. PLoS ONE 9: 1-9.

Matthey, R. 1931. Chromosomes de sauriens: Helodermatidae, Varanidae, Xantusiidae, Anniellidae, Anguidae. Bull. Soc. Vaud. Sci. Nat. 57: 269-270.

Patawang, I. and Tanomtong, A. 2017. Constitutive heterochromatin observed on metaphase chromosome of Varanus bengalensis by C-banding and DAPI methods. Cytologia 82: 1-2.
Pianka, E. R. 1995. Evolution of body size: Varanid lizards as a model system. Am. Nat. 164: 398-414.

Porter, C. A., Haiduk, M. W. and Queiroz, K. D. 1994. Evolution and phylogenetic significance of ribosomal gene location in chromosomes of squamate reptiles. Copeia 2: 302-313.

Rooney, D. E. 2001. Human Cytogenetics: Constitutional Analysis. Oxford University Press, Oxford.

Sangpakdee, W., Phimphan, S., Tengjaroenkul, B., Pinthong, K., Neeratanaphan, L. and Tanomtong, A. 2017. Cytogenetic study of tree microhylid species (Anura, Microhylidae) from Thailand. Cytologia 82: 67-74.

Singh, L., Sharma, T. and Ray-Chaudhu, S. P. 1970. Chromosome numbers and sex chromosomes in few Indian species of amphibia and reptiles. Mammal. Chromosomes Newsl. 11: 91-94. 